\title{
Editorial : Les dynamiques contemporaines de privatisation des espaces urbains dans les villes européennes
}

Editorial. The contemporary privatisation processes of urban spaces in European cities

Jean-Michel Decroly, Christian Dessouroux et Mathieu Van Criekingen

\section{(2) OpenEdition}

Journals

Édition électronique

URL : http://journals.openedition.org/belgeo/15280

DOI : $10.4000 /$ belgeo. 15280

ISSN : 2294-9135

Éditeur :

National Committee of Geography of Belgium, Société Royale Belge de Géographie

Édition imprimée

Date de publication : 31 mars 2003

Pagination : $3-20$

ISSN : 1377-2368

Référence électronique

Jean-Michel Decroly, Christian Dessouroux et Mathieu Van Criekingen, « Editorial : Les dynamiques contemporaines de privatisation des espaces urbains dans les villes européennes », Belgeo [En ligne], 1 | 2003, mis en ligne le 01 avril 2003, consulté le 24 septembre 2020. URL : http://

journals.openedition.org/belgeo/15280 ; DOI : https://doi.org/10.4000/belgeo.15280

Ce document a été généré automatiquement le 24 septembre 2020.

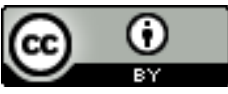

Belgeo est mis à disposition selon les termes de la licence Creative Commons Attribution 4.0 International. 


\section{Editorial : Les dynamiques contemporaines de privatisation des espaces urbains dans les villes européennes}

Editorial. The contemporary privatisation processes of urban spaces in European cities

Jean-Michel Decroly, Christian Dessouroux et Mathieu Van Criekingen pavillonnaire de la municipalité voisine, Cuincy. La grille, 2,20 mètres de haut pour 7 mètres de large, exactement la largeur de l'allée, a été installée quelques jours plus tôt par la mairie de Cuincy à hauteur de la frontière municipale, arguant qu'elle protégerait les propriétaires des pavillons des «incivilités » attribuées aux habitants de la cité HLM voisine. Pour ces derniers, en plus du sentiment d'humiliation et de colère, atteindre l'école, le collège, le marché ou le cimetière requiert désormais un détour de plus d'un kilomètre. par « des arcades de bois fleuries et un système de plots rétractables» (Le Figaro, 29 août 2002).

Depuis une dizaine d'années au moins, de telles références à des logiques d'enclavement ou d'enclosure de portions d'espaces urbains se sont multipliées. Elles nourrissent un ensemble de discours selon lesquels, principalement sur le modèle nord-américain, les villes européennes verraient proliférer, au son des plaidoyers sécuritaires venus d'outre-Atlantique (Wacquant, 1999), quartiers, communautés ou territoires privatisés, y annonçant la disparition prochaine de tout espace public au sens classique du terme. Aux Etats-Unis, les travaux de M. Davis (1997), basés sur le cas 
de Los Angeles, dépeignent en ce sens l'émergence d'un nouveau modèle urbain, la "fortress city", composée d'une myriade d'enclaves fortifiées, repliées sur ellesmêmes et dans lesquelles les classes aisées vivent et consomment sous l'œil vigilant de multiples caméras de surveillance tandis que, tout autour, les populations pauvres restent confinées dans des espaces clairement délimités et activement contrôlés. M. Davis parle à ce propos de "militarization of public space ", processus exacerbant des phénomènes de fragmentation et de ségrégation sociale qui s'intensifient. Ce type de discours en rejoint d'autres, notamment ceux centrés sur la vision d'une " revanchist city » imprégnée de tolérance zéro (voir les travaux de N. Smith, 1996, centrés sur le cas de New York), de même que les commentaires foisonnant sur la multiplication, aux Etats-Unis comme dans d'autres parties du monde (Amérique Latine, Asie p.ex.), de gated communities (p.ex. Flusty, 1997) ou de nouveaux espaces d'apparence publique mais largement privatifs dans les faits, les nouveaux malls commerciaux notamment (p.ex. Chivallon et al., 1998 ; Mermoz, 2002).

6 Ces discours partagent le constat d'une atomisation accrue des territoires urbains, desquels les espaces publics traditionnels, places, rues ou parcs publics, seraient en passe de disparaitre au profit d'une constellation d'enclaves aisées exclusivement dévolues à la consommation ou à l'entre-soi de leurs usagers, baignés dans un environnement apprivoisé, sécurisé et réservé à un public rigoureusement normé (p.ex. Meurer, 1994 ; Burniat et Genard, 2002).

7 S'il est indéniable que les espaces urbains sont aujourd'hui transformés par une série de dynamiques nouvelles, pas ou peu présentes durant la période de croissance fordiste d'après-guerre, les discours évoqués ci-dessus posent néanmoins question. En effet, la diffusion de la notion de privatisation des espaces urbains s'opère à la faveur d'un grand flou sémantique : d'une analyse à l'autre, «espaces publics » et " privatisation » sont employés pour caractériser différents types d'espaces, encourant une grande variété de transformations eu égard à des fonctions urbaines différentes et à des échelles géographiques variables. La plupart des travaux s'appuient ainsi sur une définition large et ambiguë de la notion de privatisation des espaces publics, mêlant les questions relatives au statut juridique du sol à celles portant sur l'accès à ces espaces et à leur usage. En somme, cette notion paraît constituer un "fourre-tout ", une unité artificielle d'événements différents réunis dans un même tout. En retour, cette nature "fourre-tout " permet une utilisation très extensive du concept de privatisation des espaces urbains. De nombreux auteurs alimentent de la sorte, au départ de contextes urbains très différents, un foisonnement discursif autour de cette notion. Ce foisonnement pose d'autant plus question qu'il s'appuie sur des monographies portant sur des cas emblématiques dont on ne mesure pas, faute de statistiques pertinentes, la représentativité.

\section{De multiples formes de la privatisation des espaces urbains}

Résidentialisation, communautarisation, multiplication des private neighbourhoods ou des gated communities, aménagement de résidences sécurisées, de rues ou de clos privés, création de shopping malls, d'urban entertainment centres, d'enclaves touristiques ou d'autres espaces de consommation privatifs d'allure publique, essaimage de dispositifs de technosur-veillance ou de prévention situationnelle... la notion de privatisation des 
espaces urbains est mobilisée dans une grande variété de cas, en regard de processus de natures très différentes qui s'entremêlent dans les paysages contemporains des villes. Percer le flou sur les limites et l'étendue des différents processus en jeu appelle à des efforts de différenciation et de catégorisation empiriques, peu présents à ce jour dans la littérature face à l'abondance des approches principalement discursives.

Cette perspective articule entièrement le propos de C. Dessouroux (2003). L'exploration de la notion de privatisation y est fondée sur une réflexion préalable sur le concept d'espace public dans le cadre urbain. Trois dimensions structurantes des espaces publics, autour desquelles se joue la privatisation, sont distinguées: le statut de propriété des espaces (propriété publique, privée, collective, copropriété...), l'accès à ces espaces (plus ou moins universel, soumis à différents paramètres tels que droit d'entrée, barrières physiques ou sociologiques...) et la nature des règles et normes qui en conditionnent l'usage (régulations formelles institutionnalisées, informelles de fait, plus ou moins contraignantes...). Ces trois dimensions constituent autant de paramètres qui, en se modifiant, transforment la nature des espaces concernés, indiquant de la sorte différents types de privatisation. L'article aboutit ainsi à proposer une grille d'analyse typologique sur base de laquelle la diversité des processus de privatisation en cours dans les villes occidentales peut être évaluée.

La mise en oeuvre des instruments proposés par C. Dessouroux conduit à constater que la privatisation de l'espace urbain relève moins d'une extension du domaine privé (au sens juridique du terme) au détriment du domaine public, ou du transfert vers le secteur privé du droit d'exploitation ou d'usage d'un espace public, que de l'apparition de nouvelles formes de régulation au sein tant des espaces publics (c'est-à-dire du domaine public) que des espaces privés ouverts au public (complexes commerciaux, infrastructures scolaires...) ou des espaces privés d'allure publique (espaces collectifs associés à un ensemble de logements ou d'hébergements touristiques par exemple). Ces nouvelles formes de régulation portent simultanément sur l'accès et l'usage de tels espaces. Elles interpellent les citoyens soit parce qu'elles remettent en cause certaines caractéristiques inhérentes au domaine public, soit parce qu'elles radicalisent la différence entre domaines public et privé.

11 La suite du présent article vise à offrir une vue d'ensemble sur ces modes nouveaux de régulation des espaces urbains, en distinguant domaine public et domaine privé. Dans chaque cas, nous veillons à identifier les facteurs qui surdéterminent l'apparition de ces modes de régulation, nous nous interrogeons, par comparaison avec la situation observée en Amérique du Nord, sur leur singularité au sein des villes européennes et nous examinons leurs enjeux politiques dans le cadre de la gouvernance urbaine.

\section{Les dynamiques de privatisation de l'espace public}

\section{La naissance du concept d'espace public}

12 Encore relégué au rang d'espace fonctionnalisé, résiduaire et segmenté en unités de gestion discrètes (la voirie, les réseaux techniques, l'éclairage, les plantations, les espaces verts) durant les années 1960, l'espace public a acquis depuis une trentaine d'années le statut d'un espace aux caractéristiques spécifiques, pourvu de sens et de reconnaissance et devenu un des vecteurs privilégiés d'expression des politiques urbaines. L'usage d'un terme unique, l'«espace public», qui se substitue dans les 
années 1970 à des termes tels que "espace extérieur ", "espace ouvert ou «espace collectif », souligne cette intention de manipuler désormais l'espace public comme une unité d'analyse ou d'aménagement entière, intégrant des considérations d'ordre spatial et social. Les villes de Bologne (années 1970), Barcelone (années 1980) et Lyon (années 1990) figurent parmi les villes les plus emblématiques de cet enthousiasme avoué pour l'espace public. Elles ont été suivies par de nombreuses autres villes européennes qui se sont engagées dans de vastes projets de révision de leurs espaces publics, le plus souvent dans le cadre d'opérations de restructuration de quartier et de valorisation du cadre de vie. Ces projets ont en commun de prévoir des aménagements et des règles destinés en même temps à valoriser les espaces publics et à en réglementer les usages.

\section{L'impensé de l'urbanisme}

13 L'urbanisme d'après-guerre et surtout les réalisations et projets des années 1960 ont été démesurément imprégnés de positions privilégiant les fonctions urbaines et l'économie - les deux étant étroitement liés par un esprit de rationalisation économique et de fonctionnalisation spatiale de la réalité urbaine. L'espace public est resté «l'impensé de l'urbanisme progressiste moderne » (Levy, 1996, p. 57) et se voyait réduit à une unité de planification conçue en fonction de normes techniques de mobilité et de flux (en optant pour la voiture comme mode de déplacement privilégié). Cette vision économico-fonctionnelle et la pression exercée par les fonctions de centralité de la ville ont entraîné l'apparition de voies-express (trémies, autoponts, échangeurs) et d'aires de stationnement transformant profondément le tissu d'espaces publics urbains centraux, ainsi que le développement, aux marges de la ville, de nouveaux équipements collectifs et ensembles résidentiels desservis par de grandes infrastructures routières. L'espace laissé libre par les constructions était essentiellement dévolu aux fonctions hygiénistes, récréatives et circulatoires, toutes subordonnées aux fonctions résidentielles et productives de la ville. Les espaces publics résistèrent mal à cette subordination de leurs usages coutumiers à l'impératif de la fluidité et du stationnement automobile et au morcellement fonctionnel de l'espace urbain (Voisin, 2001). La construction de lieux publics pourvoyeurs d'identité collective ou offrant la possibilité d'interactions sociales spontanées ne faisait l'objet d'aucune préoccupation sérieuse de la part des urbanistes. Les seuls espaces échappant vaguement à cette logique étaient les zones à caractère historique - converties en îlots de patrimonialisation - ou commercial, les deux étant transformées progressivement en zones piétonnes depuis les années $1950^{1}$ (Monheim, 1980).

\section{D'un enjeu des luttes urbaines...}

Porté par la vague contestataire de la fin des années 1960, un revirement dans le discours et l'action sur l'espace public s'annonça. Les luttes urbaines en pleine effervescence (Cherki \& Mehl, 1976; Mayer, 1997) remirent en cause les projets urbanistiques gargantuesques. Elles s'insurgèrent contre le fait d'avoir fait fi de l'avis des personnes les plus concernées par les politiques urbaines, les habitants de la ville, et d'avoir abouti à un état désolant de l'environnement urbain, qui ne suscitait guère d'enthousiasme. La montée en puissance des divers mouvements associatifs (de nature urbano-populaire, environnementaliste, patrimoniale...) et leur implication graduelle dans le processus décisionnel portèrent les multiples revendications sur l'espace public 
au-devant des discussions sur le devenir des villes. Ces préoccupations pour la défense $\mathrm{du}$ milieu urbain furent soutenues par des initiatives plus globales, telle que la campagne européenne pour la "Renaissance de la Cité » organisée en 1981 par le Conseil de l'Europe et qui évoquait le rôle déterminant de la reconstruction des espaces publics pour la mise en œuvre de la réhabilitation/requalification urbaine.

Le contexte démographique (suburbanisation massive), économique (désindustrialisation, problèmes financiers des centre-villes) et social (accentuation de la polarisation socio-spatiale) des années 1970 invita par ailleurs les acteurs urbains à réviser certains attributs propres à la vision urbanistique fonctionnaliste, inconciliables avec la nouvelle donne urbaine. Ce retournement de tendance souscrivait à un souci plus prononcé à l'égard du développement durable de la ville et à la genèse de nouvelles politiques urbaines qui prennent garde à intégrer entre autres des considérations d'ordre écologique (protection des espaces semi-naturels), patrimonial (conservation de paysages urbains remarquables) et social (valorisation de la mixité des fonctions et des milieux sociaux).

La critique envers les pratiques urbanistiques de l'après-guerre fut également virulente au sein du corps d'architectes et d'urbanistes qui se sentaient offusqués par la mainmise des services d'ingénierie sur l'aménagement de l'espace public. Ils plaidèrent autant en faveur d'une meilleure prise en compte de l'avis des citadins, de la valeur patrimoniale de la ville historique que d'une reconstruction de la ville à une échelle plus humaine, garante de lisibilité de la trame urbaine et dispensatrice d'un attachement identitaire à la ville (Barey, 1980). Dans cette perspective, l'espace public désignait des figures urbaines traditionnelles (places, squares, rues...), produit d'un processus de sédimentation historique. Cette demande sembla d'autant plus légitime que les espaces publics, en particulier sur dalle et au pourtour des barres et tours de logements, conçus pour les zones restructurées et nouvellement urbanisées, ne parvenaient pas à satisfaire les citadins.

Ces différents éléments ont concouru à ce que l'urbanisme fonctionnaliste et les pratiques de gestion de la ville y étant associées fussent dévalorisés aux yeux de la plupart des acteurs urbains: habitants, monde associatif, responsables politiques, architectes. Perpétuer ce mode de production des espaces urbains devenait inconcevable, tant les pressions de la part des différents usagers de la ville, les impératifs de la crise financière des pouvoirs publics locaux, les conséquences sociospatiales des restructurations postfordistes, contraignaient les différents responsables à revoir les outils de leur action.

\section{... à un instrument des politiques urbaines}

Depuis trois décennies, un ensemble de transformations des structures et des modes d'organisation des sociétés occidentales se sont affermies et ont fait dire à plus d'un qu'une nouvelle phase du capitalisme, qualifiée de "postfordiste », avait pris forme. Le mode de croissance économique et de régulation sociale post-fordiste parait avant tout appuyé sur une grande flexibilité des stratégies productives des entreprises (soustraitance, réseaux de firmes...) et des modes d'organisation du travail (exigences de mobilité, succession de contrats limités dans le temps...) (Harvey, 1989a; Swyngedouw et Kesteloot, 1991). Ce nouveau contexte est à l'origine d'une série de recompositions spatiales, notamment en termes de polarisation géographique accrue des activités dans 
les métropoles occidentales, qui apparaît d'autant plus marquée dans les environnements urbains dotés de structures économiques et sociales porteuses (Lichtenberger, 1994 ; Veltz, 1996). Dans le cadre d'une mobilité accrue des capitaux, des activités et des personnes à l'échelle de marchés supranationaux, ces nouvelles conditions nourrissent le développement d'une compétition entre les territoires, particulièrement entre villes, pour attirer, fixer et/ou maintenir les investissements. Dès lors, de nouvelles politiques urbaines émergent. D’inspiration néo-libérale, elles sont focalisées en priorité sur la recherche d'une compétitivité territoriale maximale (pour l'attraction de nouvelles entreprises, de nouveaux consommateurs, de nouveaux touristes...) et intègrent dans leur mise en oeuvre une multiplicité d'acteurs privés dans le cadre de différentes structures de partenariat public-privé (Harvey, 1989b ; Jessop, 1994 ; Hall et Hubbard, 1996). La qualité de l'environnement urbain, l'image de marque internationale de la ville ainsi que les questions relatives à la sécurité, à la propreté ou à la convivialité urbaine constituent, dans ce cadre, des préoccupations de premier ordre pour les pouvoirs publics urbains (Gravari-Barbas, 1998 ; Kampschulte, 1999). Ces paramètres sont également pris en compte dans la compétition intra-métropolitaine, notamment par les politiques qui visent à promouvoir des dynamiques de réappropriation des centre-villes par les classes moyennes et supérieures. Qualité des espaces publics, convivialité urbaine ou sécurité reviennent ainsi comme des leitmotivs dans les stratégies de rehaussement de l'attractivité (résidentielle et commerciale) des centre-villes à destination des franges aisées de la population locale, très enclines, particulièrement depuis la fin de la Seconde Guerre mondiale, à délaisser les quartiers anciens au profit d'espaces périurbains socialement plus homogènes.

19 Pour mettre en œuvre ces politiques visant à rendre plus attractive les villes, les autorités urbaines peuvent mobiliser à leur propre compte une gamme élargie d'outils de planification et d'aménagement du territoire dont elles ont obtenu la maîtrise suite au transfert d'un certain nombre de compétences depuis l'échelon national vers les échelons locaux (Schneider-Sliwa, 1998).

20 Cette double configuration d'une volonté de produire des espaces urbains attrayants et d'une maîtrise dorénavant plus autonome des outils de gestion et d'aménagement propulse l'espace public au rang d'outil de développement urbain local quasi incontournable. En tant qu'espace ouvert au regard de celui qui fréquente la ville au quotidien ou au gré d'une visite, il est un élément essentiel dans la recherche d'attractivité soutenue de la ville. Cette démarche s'inscrit dans un contexte plus large de valorisation du territoire urbain perçu par les acteurs locaux comme un enjeu très concurrentiel (Billen et al., 2002).

\section{Les nouvelles vertus de l'espace public}

21 Les espaces publics sont désormais reconnus comme élément essentiel du paysage urbain. Ils ne sont plus considérés uniquement comme des espaces d'accompagnement destinés à parfaire la finition d'un immeuble ou d'une opération urbanistique, mais sont désormais pourvus d'une fonction : celle de donner une certaine unité et identité à la ville, de produire une cohérence visuelle et fonctionnelle entre les différentes structures urbaines et de procurer à la ville une scène à l'épanouissement collectif. En substance, l'espace public offrirait un remède prometteur aux effets de la période d'urbanisation antérieure et inviterait à recréer la ville: harmoniser les fragments 
urbains disparates et cloisonnés, rétablir ou préserver la cohésion sociale, offrir des lieux de rassemblement et soutenir l'animation urbaine. Les opérations de réhabilitation de l'espace public sont ainsi révélatrices d'un postulat courant, selon lequel l'amélioration de la forme urbaine aurait des effets bénéfiques sur le tissu social.

Un des éléments les plus emblématiques de cette évolution est l'appui progressif du caractère festif et évènementiel de l'espace public sous la forme de spectacles et de divertissements en tout genre. Leur production est le fruit d'une synergie entre une demande d'animation croissante de la part des usagers de l'espace et les bienfaits en termes d'attractivité et d'image de marque escomptés par les initiateurs de telles festivités publiques.

L'origine de ces initiatives remonte aux années 1960, au cours desquelles sont apparues de nouvelles formes d'expression artistiques, qualifiées d'animations et relayées dans les années 1970 par les "arts de la rue" (Chaudoir, 2000). Dès cette époque se développe également l'idée d'intégrer ces manifestations culturelles et festives dans des structures plus formelles d'intervention culturelle dans l'espace public. Cette ambition est d'ailleurs contemporaine des diverses formes de requalification des espaces urbains centraux. L'équipement culturel s'assigne dans ce cadre un rôle majeur dans la production d'espaces valorisés (Crewe \& Beaverstock, 1998 ; Zukin, 1998). Leur succès découle également du fait que, pour une part croissante de la population, le temps de loisir ne figure plus comme simple négatif du temps de travail, mais représente désormais un espace de temps à consacrer à des activités extraprofessionneles et extra-domestiques. En réponse à cet engouement renforcé de la population à s'investir dans des espaces animés, l'adaptation des espaces urbains à cette demande devient un enjeu de marketing important. C'est ainsi que les gestionnaires d'espaces privés ouverts au public, tels les shopping malls ou multiplex de cinéma de nouvelle génération, incorporent une composante de loisirs et d'animation (par exemple l'organisation de brocantes ou d'un marché de Noël, l'installation de restaurants thématiques et de game centers...) dans leurs infrastructures pour en faire un lieu de fréquentation qui dépasse le temps des achats ou la durée du film.

Dans le cas des espaces publics urbains, la recette est semblable. Dans l'espoir de captiver l'attention de visiteurs potentiels, les villes organisent en leur sein un nombre croissant d'activités culturelles et divertissantes et ce notamment dans l'espace public. De multiples concepts qui ont fait preuve de leur efficacité dans une ville, en raison d'une réception favorable auprès de la population ou d'un écho positif dans la presse (inter)nationale, sont souvent transposés dans d'autres villes et témoignent du caractère interchangeable et utilitaire de telles initiatives. Il en est ainsi des multiples "Carnaval des cultures ", "Love Parade " et "Gay Pride " qui figurent désormais à l'agenda culturel de nombreuses villes européennes, au point de devenir un enjeu commercial de prime importance, dans la mesure où leur absence priverait les hôteliers et restaurateurs de retombées économiques essentielles. Le concept "Paris-plage » initié en 2002 à Paris constitue un exemple comparable d'usage de l'espace public. Il s'agit d'une opération d'animation très médiatisée, qui s'approprie pendant quatre semaines estivales l'espace des berges de la Seine à hauteur des îles de la Cité et de Saint-Louis, d'habitude envahi par la circulation automobile, pour offrir à leurs visiteurs toute la gamme de plaisirs de vacances à la mer sans devoir quitter la ville. L'initiative a remporté un important succès autant auprès des habitants de Paris que 
des nombreux visiteurs et touristes. La formule a depuis lors été reprise en 2003 par Bruxelles sous l'intitulé « Bruxelles-les-Bains ».

L'exposition à ciel ouvert «Cow Parade» ou «Art on Cows» a fait l'objet d'une évolution similaire. L'idée est née à Zurich en 1998 d'une envie de promouvoir l'art urbain et de donner un appui au commerce dans la ville. Le principe est simple. Des vaches en plastique moulé sont vendues vierges à des sponsors, généralement des restaurateurs, commerçants, banques ou entreprises locales, qui les font ensuite "décorer» par des artistes librement choisis. Les oeuvres animalières sont alors réparties dans les lieux visibles et fréquentés de la ville. A la fin de l'exposition, le troupeau de vaches est vendu aux enchères et l'argent est réparti entre les différents intervenants et le propriétaire du concept "Cow Parade ». Le franc succès remporté par cette initiative, l'impact appréciable sur le commerce local et les réactions d'étonnement et de plaisir qu'évoquent les visiteurs, ont poussé d'autres villes à tenter l'expérience, comme Chicago en 1999, Luxembourg en 2001 et Bruxelles en 2003.

Dans le même temps, l'espace public fait l'objet de nouvelles formes de régulation, qui matérialisent aux yeux de ses divers usagers le credo de la gouvernance urbaine néolibérale, notamment en matière de sécurité. Les articles de B. Belina (2003) et J. Massey (2003) apportent un éclairage incisif sur cette évolution. En effet, ils mettent en lumière l'intégration croissante des interventions des autorités urbaines sur les espaces publics au sein de dispositifs de "sécurisation » et de "contrôle " accru de territoires ciblés, qu'il s'agisse «d'évincer les indésirables » de quartiers anciens en redéveloppement commercial (Massey) ou d'espaces focalisant un réinvestissement public-privé à des fins de rehaussement de la compétitivité urbaine (Belina). Les exemples mentionnés par ces deux auteurs se retrouvent dans nombre d'autres villes, à Bruxelles notamment.

Les interventions actuelles des pouvoirs publics bruxellois en matière de rénovation urbaine accordent en effet une place de premier plan aux opérations sur les espaces publics (propreté, verdurisation, réfection de voiries, amélioration de l'éclairage public...) et à l'amélioration des équipements de quartier (création de maisons de jeunes, aménagement de terrains de sport...), notamment dans les quartiers les plus pauvres et dégradés du centre de la ville. Ces interventions présentent l'avantage d'être relativement légères, de pouvoir être réalisées dans des délais assez courts, de répondre à certaines attentes immédiates des habitants des quartiers concernés (organiser des collectes d'encombrants, limiter certaines nuisances liées au trafic automobile, créer des infrastructures de loisirs...) et permettent de marquer la présence de l'Etat dans des quartiers longtemps délaissés par les pouvoirs publics. Cependant, on peut également y lire la matérialisation d'une nette volonté de "sécurisation" et de "contrôle social» accru de ces quartiers. Le thème de la «sécurisation» figure en effet au cœur des dispositifs qui accompagnent les programmes de rénovation urbaine dans ces quartiers, les "Contrats de sécurité et de société » en particulier. Mis en place au début des années 1990 dans la foulée d'incidents qualifiés "d'émeutes urbaines" qui avaient secoué plusieurs quartiers populaires du centre de la ville, ce programme, dont les principes d'action sont élaborés à l'échelle fédérale (Ministère de l'Intérieur), développent une double perspective, à la fois policière (développement des missions de proximité, acquisition de nouveaux équipements, installation de caméras et de systèmes de télépolice dans certains commerces...) et de " prévention sociale » (création d'antennes de justice dans les quartiers dits "à risques", organisation d'activités sociales diverses, 
réaménagement de parcs...). De même, les contrats mis en place en 2000 dans sept communes bruxelloises dans le cadre de la politique fédérale des grandes villes mettent particulièrement l'accent sur la question de «l'insécurité ». Ces dispositifs prévoient notamment la mise à l'emploi d'allocataires sociaux dans le cadre des «nouveaux métiers de la sécurité " (agent de Prévention et de Sécurité, agents d'ambiance, stewards urbains, gardiens de parcs...) dont la finalité principale est d'investir, pour le compte de l'Etat, l'espace public (Defraene et al., 1997 ; Decroly et al., 2000; Thibaut, 2000).

28 A la faveur de la multiplication de ces dispositifs publics imprégnés de préoccupations sécuritaires, stigmatisant des territoires dits «sensibles " (et plus encore certaines franges de leur population) et y développant la techno-surveillance, le contrôle policier et la (re)mise à l'emploi par le biais de "petits boulots » à court terme, les nouveaux modes de gouvernance urbaine néo-libérale légitiment un modèle d'atomisation des collectivités et des territoires urbains.

\section{Les dynamiques de privatisation des espaces privés ouverts au public : le cas des nouveaux complexes commerciaux}

La volonté de "sécurisation" et de "contrôle social» accru dont font preuve les autorités urbaines à l'égard des espaces publics de certains quartiers imprègne aussi avec force les propriétaires et/ ou gestionnaires d'espaces privés qui, comme les complexes commerciaux, sont ouverts au public, sans droit d'entrée (Maitland, 1985 ; Davis, 1997). Les shopping malls de nouvelle génération sont ainsi fréquemment aménagés sous le mode de la "prison panoptique » de Jeremy Bentham, de façon à créer un sentiment de sécurité chez les commerçants et les chalands. En leur sein, les circulations s'effectuent donc sous un double contrôle, rendu expressément visible, de dispositifs de vidéo-surveillance - parfois complétées par de systèmes de reconnaissance faciale - et de vigiles privés. Si ces derniers sont là pour rassurer les chalands, ils ont également pour mission de préserver une atmosphère propice à la consommation en interdisant toutes conduites ou personnes susceptibles de perturber les chalands. Selon les propos du gestionnaire du Greengate Mall en Pennsylvanie, «We simply don't want anything to interfere with the shopper's freedom to not be bothered and have fun " (Crawford, 1992, 23). Comme le montrent Bergenhenegouwen et van Weesep (2003) à propos du réaménagement d'une zone commerciale au centre de Rotterdam, sont particulièrement visées ici la consommation d'alcool ou de drogue, la vente de journaux pour sans-abri ou encore les attroupements de jeunes. La mise en place d'aménagements "sadiques" (Davis, 1997), comme les bancs conçus de telle manière à empêcher les sans-abri de se coucher ou les poubelles dont il est impossible de retirer le moindre objet, vise parfois à prévenir ces conduites jugées nuisibles. Il en va de même lorsque les complexes commerciaux ne comprennent ni bancs, ni toilettes publiques, ni distributeur automatique d'eau ou qu'ils sont aménagés de façon à réduire les angles morts ou autres anfractuosités où, à l'abri des systèmes de surveillance, les « indésirables » pourraient se livrer à leurs activités « illicites ». commerçantes du centre-ville, les complexes commerciaux forment donc de plus en 
plus souvent, quelle que soit leur localisation (centre-ville ou périphérie), des " cocons " sécurisés et contrôlés au sein desquels ne subsistent de l'espace public de référence que l'alignement des commerces et des pastiches d'équipements urbains (lampadaires, fontaines...).

31 Une étape supplémentaire est franchie dans la privatisation des complexes commerciaux lorsque sont mis en place des réglementations ou des aménagements qui visent à réserver leur usage à des utilisateurs exclusifs, sans recourir pour autant au cloisonnement physique. Parmi les centres commerciaux qui ont vu le jour ces vingt dernières années, certains se caractérisent ainsi par une programmation des usages, des règlements et des aménagements qui assurent une sélection des clients tout en restant théoriquement ouverts à tous les publics. Tout d'abord, ils sont habituellement occupés par des boutiques qui fournissent des produits adaptés au goût et au budget d'une clientèle disposant de revenus confortables. Ensuite, si l'indisponibilité de marchandise à la portée de personnes à faibles ressources n'y suffit pas, l'aménagement et d'une manière générale, les systèmes de signes qui sont mis en œuvre, garantissent une mise à l'écart des populations peu solvables. A titre d'exemple, on mentionnera l'absence de raccordement aux systèmes de transport public ou la présence dans les malls de panneaux de signalisation qui mentionnent que les voies de circulation ne sont pas des voies publiques, qu'elles sont réservées à l'usage des commerçants et chalands et que la permission de les utiliser peut être facilement interrompue (Crawford, 1992).

\section{Les dynamiques de privatisation des espaces privés d'allure publique}

Les espaces privés d'allure publique font également l'objet, depuis une trentaine d'années, dans les villes occidentales, de transformations substantielles qui contribuent à en modifier l'accessibilité et l'usage. De manière générale, ces transformations vont dans le sens d'une utilisation exclusive de ces espaces au profit de catégories spécifiques de population, voire d'un véritable cloisonnement. Devenues presque banales aux Etats-Unis, ces nouvelles formes de gestion et d'aménagement des espaces collectifs associés à l'habitat, se multiplient aujourd'hui en Europe.

\section{Un processus devenu massif aux Etats-Unis}

$33 \mathrm{Au}$ cours des 30 dernières années, la croissance périurbaine aux Etats-Unis s'est traduite par la multiplication d'ensembles résidentiels privés, composés d'un ensemble de maisons individuelles ou d'appartements en copropriété et gérés directement par une association de propriétaires (Homeowner Association ou HOA) (Mc Kenzie, 1994). Ces private neighbourhoods ou lotissements privés rassemblent aujourd'hui, selon les chiffres fournis par le Community Association Institute, pas loin de 40 millions d'habitants, soit près de $15 \%$ de la population étasunienne. Ils constituent donc désormais une composante importante du marché du logement, surtout dans les zones périurbaines.

34 Au sein des lotissements privés, les espaces collectifs associés à l'habitat (rues, trottoirs, parcs, terrains de jeux...) sont transformés en espaces privatifs, réservés aux seuls résidents ou visiteurs autorisés. Cette privatisation spatiale se double d'une privatisation politique. En effet, les associations de copropriétaires, à travers leurs 
organes et à l'aide des moyens prélevés auprès de leurs membres, assurent la plupart des fonctions d'une administration locale. En ce sens, comme le propose l'article de G. Glasze (2003), elles fonctionnent comme des clubs (« club economies »). Primo, les HOAs définissent, à travers le contrat de copropriété ("Covenants, Conditions and Restrictions »), le cadre réglementaire de l'aménagement et de la vie collective au sein de ces ensembles résidentiels. Ce cadre est généralement très contraignant, tant en matière d'aménagement (par exemple, interdiction de mettre des rideaux de couleurs vives aux fenêtres, restriction de la palette des teintes pour les façades ou du nombre d'espèces pouvant être plantées dans les jardins privés visibles depuis la rue, interdiction d'installer des antennes satellites ou des parkings extérieurs...) que de vie collective (par exemple prohibition des armes à feu, limitation du nombre de brocantes privées par ménage, limitation du nombre de personnes pouvant dormir dans une chambre à coucher, voire sélection des personnes admises à résider, dans les limites de la législation sur la ségrégation) (Mc Kenzie, 1994; Thill, 2002). Secundo, les associations de propriétaires assurent diverses fonctions de gestion locale, qui vont de l'entretien de la voirie ou la collecte des immondices à l'aménagement d'infrastructures récréatives, culturelles voire scolaires (Mc Kenzie, 1994).

Le financement des HOA's est intégralement pris en charge par les copropriétaires. En retour, ces derniers s'arrogent le bénéfice exclusif des infrastructures et services fournis. Lorsque l'association de copropriétaires offre une large gamme de services, les résidents se montrent parfois soucieux d'échapper à la fiscalité locale ou d'obtenir la possibilité de déduire de leurs taxes foncières les frais payés à une HOA pour les services qu'elle rend (Kennedy, 1995 ; Le Goix, 2001). Dans ce cas, la privatisation des fonctions de gestion locale menace un système redistributif déjà malmené par plusieurs réductions successives d'impôts locaux ou fédéraux.

G. Glasze (2003) montre que le modèle de «club economies » peut aussi s'appliquer à une série de municipalités "traditionnelles", en particulier dans le cas de municipalités suburbaines des Etats-Unis composées d'une population aisée et fort homogène, et inscrites dans un contexte de large autonomie de financement des collectivités locales.

Une dernière étape dans la privatisation des espaces privés d'allure publique est franchie avec le cloisonnement. Aux Etats-Unis, dans environ 20 à 30.000 lotissements privés, rassemblant plus ou moins $20-25 \%$ de la population de ces quartiers, soit environ 10 millions de personnes, la privatisation des espaces collectifs se double d'une véritable fermeture de l'ensemble résidentiel par installation de clôtures, de barrières ou de murs d'enceinte, doublés éventuellement d'un contrôle des entrées par des agents de sécurité privée (Blakely \& Snyder, 1997; Le Goix, 2001). Dans ces gated communities, les copropriétaires sont obligés de participer financièrement à la réalisation de murs communs et d'accepter les conditions de comportement qu'ils impliquent.

\section{Une timide percée en Europe}

Comme le souligne l'éditorial d'une récente livraison de la revue Environment and Planning B consacrée aux gated communities, les private residential neighbourhoods sont encore très peu nombreux en Europe (Webster et al., 2002). En absence de données 
systématiques ou d'études empiriques de synthèse, l'évaluation de l'ampleur du phénomène reste une utopie et il faut se contenter de quelques indications sommaires.

Le principe de copropriété existe depuis plusieurs décennies en Europe. Son application est toutefois restée longtemps confinée aux immeubles construits dans les centres urbains, au sein de "copropriétés verticales». Celles-ci se distinguent nettement des private neighbourhoods tant par la modestie des surfaces collectives couvertes que par le nombre restreint des équipements co-gérés. Les espaces communs se limitent le plus souvent à une cour, aux escaliers et aux couloirs d'un immeuble. Les syndicats de copropriétaires, quant à eux, assurent uniquement l'entretien des parties communes (ravalement, réfection de toiture, nettoyage, consommation d'électricité des parties communes...) et le fonctionnement usuel des services collectifs et des éléments d'équipement commun (ascenseur, chauffage collectif, vide-ordures, interphone, antenne de télévision). Depuis une vingtaine d'années, dans le cadre du développement de lotissements périphériques, sont apparus de nouvelles formes de copropriétés, dites "horizontales". Celles-ci s'apparentent davantage aux private neighbourhoods dans le sens où le syndic y est chargé de gérer les équipements collectifs (espaces verts autres que privatifs, voiries, réseaux, bâtiments collectifs, piscine, tennis, aires de jeux...). D'après les renseignements fournis par la presse immobilière, elles prendraient quelque importance, notamment en France. Faute de données plus précises, il est toutefois impossible, en l'état actuel, de quantifier leur nombre et leur répartition spatiale.

Parce qu'elle est plus visible et plus sujette à débat, la diffusion des gated communities est mieux documentée (Jaillet, 1999 ; Glasze, 2001 ; Webster, 2001 ; Madoré, 2003). En Europe, les espaces résidentiels fermés se rencontrent avant tout dans les espaces balnéaires de l'Europe du sud, tant en Algarve, que sur les côtes espagnoles, en particulier la Costa del Sol, et la Côte d'Azur. Mais ils s'observent également dans les périphéries de quelques agglomérations non touristiques. Ainsi, dès la fin des années 1970, S. Berlusconi fit fortune en construisant un vaste ensemble de résidences fermées, destiné à 6500 personnes aisées, à Basiglio, dans la banlieue de Milan. De manière moins spectaculaire, se construisent désormais des petits ensembles résidentiels clos dans les grandes agglomérations comme Berlin, Londres, Lisbonne, Madrid ou Vienne. En France, comme le montre Madoré (2003), les programmes immobiliers " sécurisés » sont apparus dans l'agglomération de Toulouse à l'initiative d'un promoteur immobilier local. Ils ont ensuite rapidement diffusé dans les autres régions métropolitaines, en particulier dans la région parisienne. Les espaces résidentiels fermés ou clos existent également en Belgique, à l'image du tout récent projet « Hyde Park Résidence » qui vise à reconvertir un hôpital désaffecté situé dans le sud aisé de la Région de Bruxelles-Capitale en 70 logements de haut standing, au coeur d'un domaine de $17000 \mathrm{~m}^{2}$ entièrement clôturé. Ils restent pourtant largement méconnus. En dehors du cercle restreint des promoteurs immobiliers et des architectes spécialisés dans la production de l'habitat « sécurisé », qui peut dire combien de clos ont été aménagés en Belgique et quelles sont leurs caractéristiques?

41 En regard des gated communities étasuniennes, les ensembles résidentiels clos dénombrés en Europe, se distinguent à la fois par une taille modeste (rarement plus de 20 logements) et un nombre restreint d'équipements. L'accent y est mis davantage sur les dispositifs de sécurité que sur la qualité des espaces collectifs. Enfin, ils ne sont pas encore le théâtre de velléités sécessionnistes. 

dans le cadre de certains processus de gentrification résidentielle. En effet, ceux-ci s'accompagnent parfois de la fermeture plus ou moins complète et permanente d'espaces publics attenants aux ensembles résidentiels réinvestis, par exemple certaines ruelles ou impasses bordées de part et d'autre de maisons rénovées.

\section{Les logiques multiples de la privatisation des espaces privés d'allure publique}

L'émergence d'espaces privés d'allure publique sécurisés, exclusifs ou cloisonnés s'inscrit, au même titre que les nouvelles formes de régulation des espaces publics, dans la transition vers le post-fordisme. De manière générale, les nouvelles politiques urbaines centrées sur la compétitivité territoriale encouragent ces pratiques, car elles peuvent stimuler l'attractivité des villes auprès de catégories solvables de résidents et, par conséquent, fournir de nouvelles recettes fiscales, tout en reportant l'ensemble des coûts d'urbanisation sur le secteur privé.

Par ailleurs, le démantèlement du compromis fordiste-keynesien à la faveur de la crise du fordisme et de la montée en puissance de la flexibilité, a généré une nouvelle tendance à la polarisation sociale, entre les exclus ou travailleurs précaires, les salariés qualifiés survivants de la relation salariale fordiste et les nouvelles catégories aisées qui occupent les postes hautement qualifiés et fortement rémunérés dans les activités du tertiaire de pointe. De manière simplifiée, ces deux derniers groupes ont nourri une demande nouvelle pour des espaces privatisés car ils étaient touchés de près ou de loin par quatre processus concomitants : l'accroissement de leur sentiment d'insécurité, le développement du communautarisme, un attachement réaffirmé à leurs biens immobiliers et la recherche d'un cadre de vie « idéal » conforme à leur position sociale. L'analyse du cas états-unien est particulièrement instructive à cet égard.

En dépit du recul des chiffres de la criminalité observé depuis le milieu des années 1970 (Wacquant, 1999), la population des Etats-Unis n'a jamais été autant préoccupée par la sécurité des personnes. Ainsi, depuis 1993, elle a mis, pour la première fois, la peur de la criminalité au premier rang de ses préoccupations (Body-Gendrot, 1998). Largement alimenté par la mise en spectacle de la violence par les médias, ce sentiment d'insécurité est particulièrement vif chez les habitants des banlieues où il est favorisé soit par l'exagération et la méconnaissance des faits (Staszak, 1999), soit par l'hétérogénéisation récente de certains quartiers. Dans ce climat de psychose généralisée, les membres de la classe moyenne ou des catégories aisées ont de plus en plus l'impression qu'eux-mêmes et leurs biens sont menacés et que les autorités publiques sont incapables d'apporter une réponse satisfaisante à cette menace. Par ailleurs, les questions de sécurité peuvent être à l'origine d'une contagion, à l'échelle locale, des communautés encloses (Le Goix, 2001). Les résidents de quartiers situés à proximité de ces communautés sont parfois tentés de se cloisonner à leur tour pour faire face à un report - effectif ou perçu - des actes délictueux en dehors des enceintes des gated communities.

46 Au-delà de son lien étroit avec les angoisses sécuritaires, la privatisation de l'espace résidentiel consacre les aspirations «communautaires » d'une partie de la population états-unienne. En effet elle offre la possibilité à des individus partageant la même 
origine ethnique, la même tranche d'âge ou le même statut social de s'approprier intégralement un territoire au sein duquel ils pourront développer des relations sociales internes, à l'abri de toute perturbation extérieure (Pihet, 1999).

Par ailleurs, la privatisation s'inscrit pleinement dans la logique foncière qui caractérise la société nord-américaine. Dans sa percutante analyse de la cité des anges, M. Davis (1997, p. 137) note avec humour que, comme les Siciliens dans «L'Honneur des Prizzi », les propriétaires de Los Angeles aiment leurs enfants, mais ils aiment encore plus leur patrimoine immobilier. Ce rapport intime, observable de part et d'autre des Etats-Unis, conduit à tout mettre en oeuvre pour préserver la valeur de ce patrimoine. Or, il s'avère qu'un bien immobilier situé dans une gated community ou dans une autre forme de lotissement privatisé conserve voire augmente sa valeur (Blakely \& Snyder, 1997 ; Le Goix, 2002). En effet, il est dès lors protégé des forces dépréciatives qui pourraient s'exercer sur le marché, telle qu'un accroissement de la criminalité ou même une détérioration de routes mal entretenues ou la persistance d'espaces laissés en friche. Dans cette optique, les private neighbourhoods constituent une expression extrême du syndrome nimby («not in my backyard») qui consiste pour les résidents d'un quartier à lutter contre toute forme d'externalités négatives.

Enfin, les lotissements privés et autres communautés encloses sont l'expression de l'aspiration de leurs résidents à jouir non seulement d'un logement de qualité mais encore d'un cadre de vie idéalisé alliant sociabilité, sécurité et loisirs pour former une enveloppe à la fois protectrice et douillette. En réponse à cette quête, les promoteurs immobiliers mettent en marché des ensembles résidentiels qui sont proches, dans leur conception et leur promotion, des parcs à thème (Le Goix, 2001). Une attention soutenue y est portée à la qualité des équipements, au cadre paysager et à l'ornementation architecturale. Ces soins portés à l'aménagement du cadre de vie renforcent à leur tour la volonté d'une jouissance exclusive et de sécurisation de l'ensemble résidentiel.

En Europe, les salariés qualifiés et les nouveaux groupes aisés sont porteurs d'une demande de privatisation des espaces collectifs car ils sont touchés, à des degrés divers, par les mêmes processus que ceux mentionnés ci-dessus. Ces processus s'expriment toutefois avec une intensité moindre ou selon des modalités différentes qu'aux EtatsUnis. La croissance du sentiment d'insécurité, par exemple, est moins prononcée en Europe, en particulier dans les Etats scandinaves et aux Pays-Bas. De surcroît, elle ne se traduit pas nécessairement par une remise en question des prérogatives des pouvoirs publics en tant que détenteurs du monopole de la violence légitime. De la même manière, la tendance au regroupement affinitaire et communautaire est beaucoup moins sensible en Europe. Territoire anciennement peuplé, le Vieux continent ne porte pas les héritages culturels d'une immigration massive portée, sur de brefs intervalles de temps, par des communautés très différentes sur le plan linguistique ou religieux et qui ont très tôt développé des relations sociales affinitaires. Par ailleurs, comme le souligne Madoré (2003), les équipements susceptibles de catalyser des formes micro-locales de sociabilité sont généralement beaucoup moins nombreux et plus discrets dans les lotissements privés en Europe. Enfin, avec M.C. Jaillet (1999), il convient de souligner que la densité et la force des administrations territoriales s'opposent, en Europe, à toute forme de sécession urbaine. Aux Etats-Unis, au contraire, la législation offre de nombreuses opportunités pour créer de nouvelles entités municipales ou accroître l'autonomie de celles existantes. 
50 Au-delà des facteurs responsables de la formation d'une demande nouvelle pour des espaces collectifs privatisés, il convient de souligner le rôle actif joué dans la diffusion du phénomène par les acteurs de la sécurité privée et de la promotion immobilière (voir notamment l'article de F. Madoré, 2003). Dans un premier temps, aux Etats-Unis, comme en Europe, un nombre restreint de sociétés relevant de ces deux secteurs se sont lancées, dans le cadre d'une stratégie de diversification, dans la conception et la commercialisation de produits immobiliers susceptibles de rencontrer les nouvelles aspirations résidentielles des salariés qualifiés et des groupes aisés. Face au succès rencontré, ces sociétés pionnières ont rapidement été imitées par un nombre croissant d'acteurs soucieux de s'aligner sur la concurrence. Un véritable marché de lotissements privés s'est ainsi constitué. Une des particularités de ce marché est qu'il s'auto-génère. En effet, l'illusion du danger provient autant des images de violence matraquées par les médias que de l'exacerbation des effets de démonstration des produits déjà existants.

\section{Conclusion}

51 Contrairement à ce que maints discours actuels laissent penser, le phénomène de privatisation des espaces urbains n'est pas nouveau en soi dans les villes occidentales. En Europe, on peut notamment mentionner à cet égard les créations de squares résidentiels privés de Londres, espaces fermés par des portes et accessibles aux seuls résidents, qui se multiplièrent au cours du $18 \mathrm{e}$ siècle et de la première moitié du $19^{\mathrm{e}}$ siècle (Lawrence, 1993). De même, Paris a vu l'aménagement de nombreux passages privés au cours du $19^{\mathrm{e}}$ siècle, allées de haut standing surveillées par des gardiens qui, le soir venu, fermaient les grilles qui y donnaient accès (Geist, 1982). Aux Etats-Unis, les premières clôtures résidentielles sont apparues au cours de la seconde moitié du $19^{\mathrm{e}}$ siècle afin d'isoler les rues privées des beaux quartiers de New York ou Saint-Louis.

52 En tant qu'aspect récurrent de transformations urbaines issues du contexte politicoéconomique post-fordiste, les dynamiques contemporaines de privatisation d'espaces publics urbains se différencient de ces expériences antérieures. Une massification du processus semble en effet émerger, tant au plan quantitatif (plus de villes, plus de fonctions et plus de quartiers concernés) qu'au plan qualitatif (impliquant un plus large spectre social, bien au-delà des seules élites occupant le faîte de la hiérarchie sociale). Parallèlement, la privatisation des espaces urbains se manifeste sous des formes de plus en plus variées, ce qui en complique singulièrement l'analyse.

Comme en témoignent les articles rassemblés dans ce numéro, la privatisation des espaces urbains n'est plus un phénomène marginal en Europe. Longtemps obscurcie par la complexité des modes de financement des opérations sur l'espace public ou l'opacité des acteurs impliqués dans la promotion immobilière privée, elle est de mieux en mieux cernée aujourd'hui grâce à l'opiniâtreté de quelques chercheurs qui n'hésitent pas à se plonger à bras le corps dans les archives de l'administration de l'urbanisme ou à se confronter aux sociétés immobilières. Il ressort de ces travaux pionniers que les villes du Vieux continent voient se multiplier diverses formes de privatisation qui, au-delà de leurs singularités, présentent de nombreuses similitudes avec celles observées aux Etats-Unis. Glasze (2003) et Madoré (2003) montrent ainsi que les ensembles résidentiels gérés en copropriété et les communautés encloses couvrent, de part et d'autre de l'Atlantique, un spectre social large et qu'ils s'y inscrivent dans un double processus de sécurisation et de socialisation. De surcroît, plusieurs indices laissent 
supposer que l'Europe va à son tour être gagnée par une massification de la privatisation des espaces urbains (Webster, 2001), ce qui remettrait en question nos représentations de la ville européenne comme lieu d'intégration sociale où, au sein des espaces publics, se rencontrent et se mettent en relation les multiples identités d'une société complexe.

\section{BIBLIOGRAPHIE}

BAREY A. (1980), Déclaration de Bruxelles, Editions des Archives d'Architecture Moderne, Bruxelles. BELINA B. (2003), « Evicting the Undesirables. The Idealism of Public Space and the Materialism of the Bourgeois State ", Belgeo, 1, pp. 47-62.

BERGENHENEGOUWEN G. \& VAN WEESEP J. (2003), « Manipulated space : the 'Beurstraverse' retail complex in Rotterdam », Belgeo, 1, pp. 79-86.

BILLEN C., DECROLY J.-M. \& VAN CRIEKINGEN M. (2002), « Les mutations contemporaines de la valorisation des territoires » in VANDERMOTTEN C. (éd.), Le développement durable des territoires, Editions de l'Université de Bruxelles, Bruxelles, pp. 113-130.

BLAKELY E.J. \& SNYDER M.G. (1997), Fortress America. Gated communities in the US, Washington D.C., Brookings Institution Press.

BODY-GENDROT S. (1998), Les villes face à l'insécurité. Des ghettos américains aux banlieues françaises, Bayard, Paris.

BORDREUIL S. (1993), « Hommes à la rue aux Etats-Unis. La crise des infrastructures de la ville », Les Annales de la Recherche Urbaine, 57-58, p. 135-146.

BURNIAT P. \& GENARD J.-L. (2002), « En guise d'introduction : privatisation des villes et urbanisme managérial », Les cahiers de la Cambre - Architecture, 1, pp. 4-13.

CHAUDOIR P. (2000), Discours et figures de l'espace public à travers les « arts de la rue »-La ville en scènes, L'Harmattan, Paris.

CHERKI E. \& MEHL D. (1976), « Quelles luttes ? Quels acteurs ? Quels résultats ? ^ Autrement, 6.

CHIVALLON C., MARME N. \& PROST D. (1998), « Artefact de lieu et urbanité, le centre commercial interrogé ", Les annales de la recherche urbaine, 78, pp. 28-37.

CRAWFORD M. (1992), « The World in a Shopping Mall », in M. SORKIN (ed.), Variations on a Theme Park, New York, Hill and Wang, pp. 3-30.

CREWE L. \& BEAVERSTOCK J. (1998), «Fashioning the city : Cultures of consumption in contemporary urban spaces ", Geoforum, 3, pp. 287-308.

DAVIS M. (1997), City of quartz. Los Angeles capitale du futur, La Découverte, Paris.

DECROLY J.-M., VAN CRIEKINGEN M. \& CRAHAY A. (2000), « Les conséquences socio-spatiales des politiques urbaines 'entrepreneuriales' ", Ruimte \& Planning, 20, 1, pp. 51-64.

DEFRAENE D., LALIEUX K., MARY P. \& SMEETS S. (1997), « Les Contrats de sécurité et de société dans la Région de Bruxelles-Capitale », dossier du BRES, 34. 
DESSOUROUX C. (2003), « La diversité des processus de privatisation de l'espace public dans les villes européennes », Belgeo, 1, pp. 21-46.

FLUSTY S. (1997), « Building Paranoia », in ELLIN N. (ed.), Architecture of fear, Princeton Architectural Press, New York, pp. 47-59.

GEIST J. F. (1982 ; 1989 pour l'édition française), Le passage. Un type architectural du XIX ${ }^{e}$ siècle, Pierre Mardaga, Liège.

GLASZE G. (2001), « Geschlossene Wohnkomplexe ('gated communities') als Enklaven des Wohlbefindens in der wirtschaftsliberalen Stadt », in ROGGEN-THIN H. (ed), Stadt - der Lebensraum der Zukunft? Gegenwärtige raumbezogene Prozesse in Verdichtungsräumen der Erde, Mainzer Kontaktstudium Geographie 7, pp. 39-55.

GLASZE G. (2003), « Private neighbourhoods as club economies and shareholder democracies », Belgeo, 1, pp. 87-98.

GRAVARI-BARBAS M. (1998), «Belle, propre, festive et sécurisante : l'esthétique de la ville touristique ", Norois, 45, 178, pp. 175-193.

HALL T. \& HUBBARD P. (1996), « The entrepreneurial city : new urban politics, new urban geographies ? », Progress in Human Geography, 20, 2, pp. 153-174.

HARVEY D. (1989a), The condition of postmodernity, Blackwell, Oxford.

HARVEY D. (1989b), « From managerial-ism to entrepreneurialism : the transformation in urban governance in late capitalism », Geografiska Annaler, 71b, 1, pp. 3-17.

JAILLET M.-C. (1999), « Peut-on parler de sécession urbaine à propos des villes européennes ? », Esprit, 258, pp. 145-167.

JESSOP B. (1994), « Post-fordism and the state », in AMIN A. (Ed.), Post-fordism, Blackwell, Oxford, pp. 251-279.

KAMPSCHULTE A. (1999), « 'Image' as an instrument of urban management », Geographica Helvetica, 54, 4, pp. 229-241.

KENNEDY D.J. (1995), « Residential associations as state actors: Regulating the impact of gated communities on non-members », Yale Law Journal, 105, 3, pp. 761-793.

LAWRENCE H. W. (1993), «The greening of the squares of London : transformation of urban landscapes and ideals ", Annals of the association of American geographers, 83, 1, pp. 90-118.

LE GOIX R. (2001), « Les communautés fermées dans les villes des Etats-Unis. Aspects géographiques d'une sécession urbaine ", L'espace géographique, 1, pp. 81-93.

LE GOIX R. (2002), « Les gated communities à Los Angeles, place et enjeux d'un produit immobilier pas tout à fait comme les autres ", L'espace géographique, 4, pp. 328-344.

LEVY A. (1996), «L'espace public de la ville méditerranéenne : mythe et réalité », in L'espace public dans la ville méditerranéenne, Actes du colloque de Montpellier 14-16 mars 1996, Editions de l'Esperou, Montpellier, pp. 57-66.

LICHTENBERGER E. (1994), « Europa und die Zukunft der Stadt. Das metropolitane Zeitalter in Europa in West und Ost », Mitteilungen der Österreichi-schen Geographischen Gesellschaft, 136, pp. 7-36.

MADORE F. (2003), « Logiques géographiques et sociales de l'enclosure des espaces résidentiels en France », Belgeo, 1, pp. 99-113. 
MAITLAND B. (1985), The New Architecture of the Retail Mail, Architecture Design and Technology Press, London.

MASSEY J. (2003), « Public Consumption : A Private Enterprise ?», Belgeo, 1, pp. 63-78.

MAYER M. (1997), « Les mouvements sociaux comme acteurs politiques dans les villes européennes : leur évolution entre les années soixante-dix et quatre-vingt-dix » in BAGNASCO A. \& LE GALES P. (dir.), Villes en Europe, La Découverte, Paris, pp. 173-200.

MC KENZIE E. (1994), Privatopia. Homeowner Associations and the Rise of Residential Private Government, New Haven and London, Yale University Press.

MERMOZ M. (2002), « Centres commerciaux. Un nouvel espace urbain », Alternatives économiques, 205, pp. 52-55.

MEURER B. (ed.) (1994), Die Zukunft des Raumes. The future of space, Campus Verlag, Frankfurt/New York.

MONHEIM R. (1980), « Fussgängerbereiche und Fussgängerverkehr in Stadt-zentren in der Bundesrepublik Deutschland», Bonner geographische Abhandlungen, 64, pp. 290.

PIHET C. (1999), « Le développement d'une territorialisation produite par l'âge : les retirement communities aux Etats-Unis », Annales de Géographie, 608, pp. 420-435.

SCHNEIDER-SLIWA R. (1998), « Städte unter neuen Rahmenbedingungen : Trends und Chancen », Regio Basiliensis, 39, 2, pp. 111-121.

SMITH N. (1996), The new urban frontier. Gentrification and the revanchist city, Routledge, London.

STASZAK J.-F. (1999), « Détruire Détroit. La crise urbaine comme produit culturel », Annales de Géographie, 609, pp. 277-298.

SWYNGEDOUW E. \& KESTELOOT C. (1991), « Le passage socio-spatial du fordisme à la flexibilité : une interprétation des aspects spatiaux de la crise et de son issue », Espaces et Sociétés, 55, pp. 243-265.

THIBAUT C. (2000), Sécurité et ville : l'influence des politiques urbaines et sécuritaires sur l'espace public, mémoire de licence, Ecole des sciences criminologiques, Université Libre de Bruxelles.

THILL S. (2002), « Learning from celebration », Les cahiers de la Cambre - Architecture, 1, pp. 59-72.

VELTZ P. (1996), Mondialisation, villes et territoires. L'économie d'archipel, Presses Universitaires de France, Paris.

VOISIN B. (2001), « Espaces publics, espaces de ville, espaces de vie » in TOUSSAINT J.-Y. \&

ZIMMERMANN M. (dir.), User, observer, programmer et fabriquer l'espace public, Presses polytechniques et universitaires romandes, Lausanne, pp. 33-47.

WACQUANT L. (1999), Les prisons de la misère, Raisons d'agir, Paris.

WEBSTER C.J. (2001), « Gated cities of tomorrow », Town Planning Review, 72, pp. 149-170.

WEBSTER C.J., GLASZE G. \& FRANTZ K. (2002), « Guest editorial », Environment and Planning B, 29 , pp. 215-320.

ZUKIN S. (1998), « Urban Lifestyles : Diversity and standardisation in spaces of consumption », Urban Studies, 35, 5-6, pp. 825-839. 


\section{NOTES}

1. P.ex. Stuttgart: 1953, Hannovre: 1956, Copenhague: 1962, Norwich: 1967.

\section{RÉSUMÉS}

Cet article introductif propose un cadrage des travaux réunis dans ce numéro. Après avoir clarifié le contenu sémantique de la notion de "privatisation » des espaces urbains, il esquisse une vue d'ensemble sur les modes nouveaux de régulation de ces espaces, en termes d'accès ou d'usage, en distinguant domaine public et domaine privé. Il cherche, dans chaque cas, à mettre en évidence les facteurs qui surdéterminent la généralisation de ces nouveaux modes de régulation, à examiner la singularité de ces processus dans les villes européennes par comparaison avec la situation observée en Amérique du Nord et à révéler les enjeux politiques soulevés par ces dynamiques dans le cadre de la gouvernance urbaine.

This article introduces the papers gathered in the issue. After an investigation of the semantic content of the "privatisation" concept, the article sketches an overall picture of the new regulation patterns of urban spaces, in terms of access and use, so much in the public as in the private realm. In each case, it seeks to find out the factors behind the generalisation of these new regulation patterns of urban spaces, questions the issue of the peculiarity of the processes of privatisation in European cities in comparison with experiences reported in the North-American urban context, and points out some political dimensions of these processes for urban governance.

\section{INDEX}

Keywords : privatisation, public urban spaces, shopping malls, housing developments, gated communities, Europe, United States

Mots-clés : privatisation, espaces urbains publics, complexes commerciaux, lotissements privés, communautés encloses, Europe, Etats-Unis

\section{AUTEURS}

\section{JEAN-MICHEL DECROLY}

Université Libre de Bruxelles, Institut de Gestion de l'Environnement et d'Aménagement du Territoire, jmdecrol@ulb.ac.be

\section{CHRISTIAN DESSOUROUX}

Université Libre de Bruxelles, Institut de Gestion de l'Environnement et d'Aménagement du Territoire, cdessour@ulb.ac.be 


\section{MATHIEU VAN CRIEKINGEN}

Université Libre de Bruxelles, Institut de Gestion de l'Environnement et d'Aménagement du Territoire, mvancrie@ulb.ac.be 